\title{
A Multistep Preparation of 3-Aryl-8-methoxythiazolo [3', 2' : 2, 3] [1, 2, 4] triazino [5, 6-b] indoles Under Microwave IR-radiations
}

\author{
RAVINDER SINGH \\ Department of Chemistry, Government College for Women, Sampla, India. \\ ${ }^{*}$ Corresponding author E-mail: gahlawat.ravinder@gmail.com \\ http://dx.doi.org/10.13005/ojc/300141
}

(Received: October 16, 2013; Accepted: November 11, 2013)

\begin{abstract}
The different 3-Aryl-8-methoxythiazolo [3', 2' : 2, 3] [1, 2, 4] triazino [5, 6-b] indoles having antihistaminic, antithyroid, antitubercular, antifungal \& antibacterial activities are synthesized through a multistep preparation in high yield in shorter reaction time under microwave irradiations.
\end{abstract}

Key words: Indole, Microwave, Aryl, heterocyclic.

\section{INTRODUCTION}

Bridgehead nitrogen heterocycles containing thiazole and related heterocycles (thiadiazole and thiadiazine) exhibit antihistaminic, antithyroid, antitubercular, antifungal \& antibacterial activities $^{1-3}$ and their synthetic importance has been greatly enhanced by the recent uses of their condensed bridgehead nitrogen heterocycles as anthelminitics, antidepressants, platelet aggregation inhibitors, antineoplastic, vulcanization accelerators and photographic sensitizers ${ }^{4-11}$. The indoles are already been synthesized by different method But they requires longer reaction time and tedious workup ${ }^{12-21}$. Microwave assisted reactions are gaining much more importance in synthetic organic chemistry due to dramatic reduction in time from days to hours and hours to minutes or seconds ${ }^{22-}$ 24.
The present work reports the synthesis of 3-Aryl-8-methoxythiazolo[3', 2' : 2, 3][1, 2, 4] triazino[5, 6-b]indoles in a multi step preparation in high yield in shorter reaction time(Scheme 1).

Ourworkstarted by reacting 6-methoxyisatin with thiosemicarbazide in Anhyd. ethanol under microwave irradiation at $560 \mathrm{~W}$ for 5 -minutes to give 6-Methoxyisatin-3-thiosemicarbanzone (I). After separation, the 6-Methoxyisatin-3thiosemicarbanzone(I) reacts with $5 \% \mathrm{KOH}$ under microwave irradiation at $560 \mathrm{~W}$ for 5 -minutes to give 7-Methoxy-5H-2,3-dihydro [1, 2, 4] triazino [5, 6-b] indole-3-thione (II).

The compound 7-Methoxy-5H-2,3-dihydro $[1,2,4]$ triazino $[5,6-b]$ indole-3-thione (II) further reacts with $\mathrm{p}$-chlorophenacyl bromide under 
<smiles></smiles>

IV
Scheme-I<smiles>COc1ccc2c(c1)[nH]c1nc(=S)[nH]nc12</smiles>

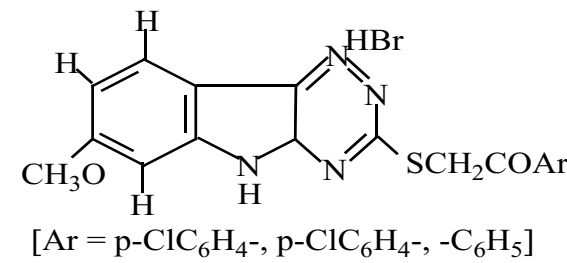

III microwave irradiation at $560 \mathrm{~W}$ for 5 -minutes to give $5 \mathrm{H}$ 3-(p-chlorophenacylthio-7-methoxy [1, 2, 4] triazino [5, 6-b] indole hydrobromide (IIla; $\mathrm{Ar}=\mathrm{p}-\mathrm{C}_{1} \mathrm{C}_{6} \mathrm{H}_{4}$ ). Similarly, 7-Methoxy-5H-2,3-dihydro [1, 2, 4] triazino [5, 6-b] indole-3-thione(II) was also irradiated with p-cholorophenacyl bromide, phenacyl bromide, under microwave irradiation at 560W for 5-minutes to give $5 \mathrm{H}-3$-(p-bromophenacylthio-7-methoxy [1, 2, 4] triazino [5, 6-b] indole hydrobromide( $\mathrm{IIlb}, \mathrm{Ar}=\mathrm{p}-\mathrm{Br}$ $\mathrm{C}_{6} \mathrm{H}_{4}$ ) and 5H-3-(phenacylthio-7-methoxy [1, 2, 4] triazino [5, 6-b] indole hydrobromide (IIla; $\mathrm{Ar}=\mathrm{C}_{6} \mathrm{H}_{5}$ ) respectively. The results are shown in Table-1.

We further explore our work by irradiated 5H-3-(p-chlorophenacylthio-7-methoxy [1, 2, 4] triazino [5, 6-b] indole hydrobromide (IIla; $\mathrm{Ar}$ $=\mathrm{p}-\mathrm{C} 1 \mathrm{C}_{6} \mathrm{H}_{4}$ ) in a mixture of $\mathrm{H}_{3} \mathrm{PO}_{4} / \mathrm{P}_{2} \mathrm{O}_{5}$ under microwave irradiation at $560 \mathrm{~W}$ for 5 -minutes to give 3-(p-chlorophenyl-8-methoxythiazolo[3', 2' : 2, 3][1, 2, 4] triazino[5, 6-b]indole(IVa, $\mathrm{R}=\mathrm{Cl})$. Similarly, 5H3-(p-bromophenacylthio-7-methoxy [1, 2, 4] triazino $[5,6-b]$ indole hydrobromide (IIIb; $\mathrm{Ar}=\mathrm{p}-\mathrm{BrC}_{6} \mathrm{H}_{4}$ ) and $5 \mathrm{H}-3-($ phenacylthio-7-methoxy $[1,2,4]$ triazino $[5,6-b]$ indole hydrobromide (IIla; $\mathrm{Ar}=\mathrm{C}_{6} \mathrm{H}_{5}$ ) were also irradiated in a mixture of $\mathrm{H}_{3} \mathrm{PO}_{4} / \mathrm{P}_{2} \mathrm{O}_{5}$ under microwave irradiation at $560 \mathrm{~W}$ for 5 -minutes to give 3-(p-bromophenyl-8-methoxythiazolo[3', 2' : 2, 3][1, $2,4]$ triazino[5, 6-b]indole(IVa, R = Br) and 3-(phenyl8-methoxythiazolo[3', 2' : 2, 3][1, 2, 4] triazino[5, 6-b] indole(IVa, $\mathrm{R}=\mathrm{H}$ ) respectively. The results are shown in Table-2.

\section{EXPERIMENTAL}

All the melting points reported are uncorrected. Infrared spectra $\left(v_{\max }\right.$ in $\left.\mathrm{cm}^{-1}\right)$ were recorded in nujol mull or $\mathrm{KBr}$ on a Perkin-Elmer 842/Beckman IR-20 / Hitachi 215 spectrometers. The proton magnetic resonance spectra were recorded on a VXR-200 MHz or R-32 Perkin-Elmer $90 \mathrm{MHz}$ spectrometer in $\mathrm{CDC1}_{3}$ or $\mathrm{DMSO}-\mathrm{d}_{6}$ using tetramethylsilane (TMS) as internal reference stadnard. The chemical shifts are expressed in $\mathrm{d}$ (ppm) units downfield from TMS. Mass spectra were scanned on a Jeol JMX-DX-300 spectrometer operating at $70 \mathrm{eV}$. Carbon, hydrogen and nitrogen analyses were carried out on a Yanaco MT-3 (JAPAN) instrument. Thin layer chromatography (TLC) were performed on silica-gel plates using acetonebenzene ( $1: 3$ or $1: 2)$ as solvent system and iodine chamber as visualizing agent.

\section{Typical procedure for the synthesis of} 6-Methoxyisatin-3-thiosemicarbanzone(I)

A mixture of 6-methoxyisatin( $0.18 \mathrm{~g}, 0.001$ $\mathrm{mol})$ in Anhyd. ethanol $(2 \mathrm{ml})$ and thiosemicarbazide $(0.1 \mathrm{~g}, 0.0011 \mathrm{~mol})$ in a mixture of water $(2 \mathrm{ml})$ and glacial acetic acid $(0.5 \mathrm{ml})$ was irradiated under microwave irradiation at $560 \mathrm{~W}$ for 5 -minutes. A yellow coloured solid formed during irradiation. The solid was filtered, washed well with water and crystallized from ethanol-DMF furnishing yellow crystals. yield $0.247 \mathrm{~g}(95 \%)$, m.p. $265^{\circ} \mathrm{C}$. [Found : N, 22.68, S, 
12.62. $\mathrm{C}_{10} \mathrm{H}_{10} \mathrm{~N}_{4} \mathrm{O}_{2} \mathrm{~S}$ requires $\mathrm{N}, 22.40 ; \mathrm{S}, 12.80 \%$ ]; IR : 825, 860 (1, 2, 4-trisubstituted benzene ring), 1115 (C=S), 1125 \& 1370 (C-O-C stretching), 1620 $(\mathrm{C}=\mathrm{N}), 1700(\mathrm{C}=\mathrm{O}), 3200,3280,3400\left(\mathrm{NH}, \mathrm{NH}_{2}\right)$.

Typical procedure for the synthesis of 7-Methoxy$5 \mathrm{H}-2,3-d i h y d r o[1,2,4]$ triazino $[5,6-b]$ indole-3thione(II)

6-Methoxyisatin-3-thiosemicarbazone (I, $0.125 \mathrm{~g}, 0.0005 \mathrm{~mole})$ in $5 \% \mathrm{KOH}(3.5 \mathrm{ml})$ was irradiated under microwave irradiation at $560 \mathrm{~W}$ for 5-minutes. The reaction mixture was cooled and the insoluble material removed by filtration. The filtrate on neutralisation with dil. $\mathrm{HCl}$ gave a yellow solid which was filtered, washed well with water and crystallised from aq. DMF furnishing yellow crystals, yield 0.108 g (92\%), m.p. > $260^{\circ} \mathrm{C}$ [Found : C, 51.91; H, 3.57; $\mathrm{N}, 23.84 ; \mathrm{S}, 13.58 . \mathrm{C}_{10} \mathrm{H}_{8} \mathrm{~N}_{4} \mathrm{SO}$ requires $\mathrm{C}, 51.72$; H, 3.45; N, 24.13; S, 13.79\%]; IR : 810, 860 (1, 2,
4-Trisubstituted benezene ring), $1150(\mathrm{C}=\mathrm{S}), 1170$, 1370 (C-O-C stretching), 1590, $1610(\mathrm{C}=\mathrm{N}), 3200$ ( $\mathrm{N}-\mathrm{H}$ stretching).

Typical procedure for the synthesis of $5 \mathrm{H}-3-(\mathrm{p}-$ chlorophenacylthio-7-methoxy[1, 2, 4]triazino [5, 6-b] indole hydrobromide (IIla; $\mathrm{Ar}=\mathrm{p}-\mathrm{C} 1 \mathrm{C}_{6} \mathrm{H}_{4}$ )

A mixture of $\mathrm{II}(0.232 \mathrm{~g}, 0.001 \mathrm{~mol})$ and p-cholorophenacyl bromide $(0.234 \mathrm{~g}, 0.001 \mathrm{~mol})$ in DMF $(6 \mathrm{ml})$ was irradiated under microwave irradiation at 560W for 5-minutes, and poured into ice-water. The solid thus separated, was filtered, washed with water and crystallized from aq. DMF to give IIla as yellow crystals, yield $0.415 \mathrm{~g}$ (94\%), m.p. > 260 [Found: N, 12.20; S, 6.92. $\mathrm{C}_{18} \mathrm{H}_{14} \mathrm{~N}_{4} \mathrm{O}_{2}$ $\mathrm{SBrCl}$ requires $\mathrm{N}, 12.03 ; \mathrm{S}, 6.87 \%]$; IR : 810, 860 (1, 2, 4-trisubstituted benzene ring), 1170, 1370 (C-O-C stretching), 1570 (C-N stretching), 1590, $1610(\mathrm{C}=\mathrm{NO}, 1690(\mathrm{C}=\mathrm{O}), 3180(\mathrm{~N}-\mathrm{H}$ stretching).

Table 1: 3-p-Chlorophenyl-8-methoxythiazolo

[3', 2' : 2, 3][1, 2, 4] triazino [5, 6-b] indole

\begin{tabular}{|c|c|c|c|c|}
\hline S. No. & Substrate(R) & Time(in minutes) & Yield(\%) & m.p. $\left({ }^{\circ} \mathrm{C}\right)$ \\
\hline 1. & $-p \mathrm{plC}_{6} \mathrm{H}_{4}-(\mathrm{IIIa})$ & 5 & 94 & $>260^{\circ} \mathrm{C}$ \\
\hline 2. & $-\mathrm{pBrC}_{6} \mathrm{H}_{4}-(\mathrm{IIIb})$ & 5 & 91 & $>260^{\circ} \mathrm{C}$ \\
\hline 3. & $-\mathrm{C}_{6} \mathrm{H}_{5}(\mathrm{IIIC})$ & 5 & 98 & $>260^{\circ} \mathrm{C}$ \\
\hline \multicolumn{5}{|c|}{$\begin{array}{c}\text { Table 2: 3-p-Chlorophenyl-8-methoxythiazolo } \\
{\left[3^{\prime}, 2^{\prime}: 2,3\right][1,2,4] \text { triazino }[5,6-b] \text { indole }}\end{array}$} \\
\hline S. No. & Substrate(R) & Time(in minutes) & Yield(\%) & m.p. $\left({ }^{\circ} \mathrm{C}\right)$ \\
\hline 1. & $-\mathrm{Cl}(\mathrm{IVa})$ & 5 & 91 & $>250^{\circ} \mathrm{C}$ \\
\hline 2. & $-\mathrm{Br}(\mathrm{IVb})$ & 5 & 90 & $>250^{\circ} \mathrm{C}$ \\
\hline 3. & $-H(I V c)$ & 5 & 96 & $>250^{\circ} \mathrm{C}$ \\
\hline
\end{tabular}

Following members of the series were also prepared in a similar way

Illb $\left(\mathrm{Ar}=\mathrm{p}-\mathrm{BrC}_{6} \mathrm{H}_{4}^{-}\right)$, yield $(91 \%)$, m.p. > $260^{\circ} \mathrm{C}$ [Found : N, 11.23; S, 6.38, $\mathrm{C}_{18} \mathrm{H}_{14} \mathrm{~N}_{4} \mathrm{O}_{2} \mathrm{SBr}_{2}$ requires, N, 10.98; S, 6.27\%]; IR: 1570 (C-N stretching), $1610(\mathrm{C}=\mathrm{N}), 1690(\mathrm{C}=\mathrm{O}), 3190(\mathrm{~N}-\mathrm{H})$. IIlc $\left(\mathrm{Ar}=\mathrm{C}_{6} \mathrm{H}_{5}\right)$, yield (98\%) m.p. $>260^{\circ} \mathrm{C}$ [Found : $\mathrm{N}, 14.62 ; \mathrm{S}, 8.18 . \mathrm{C}_{18} \mathrm{H}_{15} \mathrm{~N}_{4} \mathrm{O}_{2} \mathrm{SC} 1$ requires $\mathrm{N}, 14.49$; S, 8.27\%]; IR : 1575 (C-N stretching), $1600(\mathrm{C}=\mathrm{N})$, 3240 (N-H stretching).
Typical procedure for the synthesis of 3-pChlorophenyl-8-methoxythiazolo[3', 2' : 2, 3][1, 2, 4] triazino [5, 6-b] indole(IVa, R = C1)

Ketone $\mathrm{Illa}(0.1 \mathrm{~g})$ in a mixture of $\mathrm{H}_{3} \mathrm{PO}_{4}$ $(0.3 \mathrm{ml})$ and $\mathrm{P}_{2} \mathrm{O}_{5}(0.4 \mathrm{~g})$ was irradiated under microwave irradiation at $560 \mathrm{~W}$ for 5-minutes. The reaction mixture was poured into water and neutralised with aq. $\mathrm{K}_{2} \mathrm{CO}_{3}$ solution. The solid, thus separated, was filtered, washed well with water and crystallised from aq. DMF to furnish IVa as dark red crystals, yield $0.071 \mathrm{~g}(91 \%)$, m.p. > $250^{\circ}$ [Found : 
C, 59.17; H, 3.11; N, 15.16; S, 8.91. $\mathrm{C}_{18} \mathrm{H}_{11} \mathrm{~N}_{4} \mathrm{SOCl}$ requires $\mathrm{C}, 58.93 ; \mathrm{H}, 3.01 ; \mathrm{N}, 15.27 ; \mathrm{S}, 8.73 \%$ ]; IR; 1515 (C-N stretching) 1600 (C=N). PMR (DMSO-d $\mathrm{d}_{6}$ ) : $3.95\left(3 \mathrm{H}, \mathrm{s}, \mathrm{C}_{8}-\mathrm{OCH}_{3}\right), 7.40\left[2 \mathrm{H}, \mathrm{d}(\mathrm{J}=7.5 \mathrm{~Hz}), \mathrm{H}-3^{\prime}\right.$ and $\left.\mathrm{H}^{-} 5^{\prime}\right], 7.70$ [2H, d $(\mathrm{J}=7.5 . \mathrm{Hz}), \mathrm{H}-2^{\prime}$ and $\left.\mathrm{H}-6^{\prime}\right]$, $7.90\left(1 \mathrm{H}, \mathrm{s}, \mathrm{C}_{2}-\mathrm{H}\right), 6.9-8.1(3 \mathrm{H}, \mathrm{m}$, aromatic protons of indole moiety).

\section{Following members of the series were also} prepared in a similar way

IVb $(\mathrm{R}=\mathrm{Br})$ : yield $(90 \%)$, m.p. $>250^{\circ} \mathrm{C}$

[Found: C, 52.38; H, 2.76; N, 13.42; S, 7.51. $\mathrm{C}_{18} \mathrm{H}_{11} \mathrm{~N}_{4} \mathrm{SOBr}$ requires $\mathrm{C}, 52.55 ; \mathrm{H}, 2.67 ; \mathrm{N}, 13.62 ; \mathrm{S}$,
7.78\%]; IR : 1520 (C-N stretching), $1610(\mathrm{C}=\mathrm{N})$.

IVc $(\mathrm{R}=\mathrm{H})$ : yield (96\%), m.p. $>250^{\circ} \mathrm{C}[$ Found : C, 64.92; H, 3.68; N, 16.64; S, 9.87. $\mathrm{C}_{18} \mathrm{H}_{11} \mathrm{~N}_{4} \mathrm{SO}$ requires $\mathrm{C}, 65.06 ; \mathrm{H}, 3.61 ; \mathrm{N}, 16.86 ; \mathrm{S}, 9.63 \%]$; IR : 1520 (C-N stretching), $1610(\mathrm{C}=\mathrm{N})$.

\section{ACKNOWLEDGMENTS}

We thank Professor D. Villemin (France), Dr. R. Sharma (Dayton, USA) and Professor A.J. Bellamy (Swindon, UK) for inspiration.

\section{REFERENCES}

1. Jag Mohan, G.S.R. Anjaneyulu \& Kiran, Indian J. Chem. 27B: 128 (1988).

2. H.R. Snyder, Jr. and L.E. Benjamin, J. Med. Chem., 9: 402 (1966).

3. Norwich Pharmacal Co. Neth. Appl. 6:400,380 (1964); Chem. Abstr., 62: 2780(1965).

4. D.L. Trepanier and P.E. Krieger, U.S. Pat., 3: 641,019 (1972); Chem. Abstr., 76: 127024k (1972).

5. C.J. Sharpe, R.S. Shadbolt, A. Ashford and J.W. Ross, J. Med. Chem., 14: 977 (1971).

6. M.M. KOchhar and B.B. Williams, J. Med. Chem., 15: 322 (1972).

7. S. Kano and T. Noguchi, Japan Pat., 71: 37, 836 (1971); Chem. Abstr., 76: 25295g (1972).

8. E.E. Renfrew and H.W. Pons, U.S. Pat., 3: 950,130 (1976); Chem. Abstr., 85: 22753e (1976).

9. P.W. Jenkins and L.G.S. Brooker, U.S. Pat., 3: 681,081 (1972); Chem. Abstr., 78, $85949 z$ (1973).

10. M. Hinata, K. SHiba, H. Takei, A. Sato and T. Sakai, Ger. Offen., 2: 418, 278 (1974); Chem. Abstr., 82: 49815K (1975).

11. L.G.S. Brooker, U.S. Pat., 2: 089,729 (1937); Chem. Abstr. 31: 6989 (1937).

12. (a) Romagnoli, R; Baraldi, P.G; Cruz-Lopez, C Preti, D Bermejo, J Estavez, F. Chem. Med. Chem. 4: 1668 (2009).

(b) Bursavich, M. G Gilbert, A.M Lombardi, S
Georgiadis, K. E. Reifenberg, E Flannery, C. R; Morris, E.A. bioorg. Med. Chem. Lett. ,17: 5630 (2007).

(c) Konkel, M.J Packiarajan, M Chem. H Topiwala, U.P ${ }_{4}$ Jimenez, H Talisman, I.J Coate, H Walker, M.W. Bioorg. Med. Chem. Lett, 16: 3950 (2006).

(d) Lam, P.Y.S Vinoent, G Clark, C.G Dcudon, S Jadhav, P.K. Tetrahedron Lett, 42: 3415 (2001).

13. (a) Shindikar, A.V Khan, F Viswanathan, C.L. Eur.J.Med. Chem. 41: 786 (2006).

(b) Moser, $\mathrm{P}_{4}$ Sallmann, A Wieserberg, I.J. Med. Chem. 33: 2358 (1990).

(c) Sarges, R Howard, H. R Koe. H.K Weissman, A.J.Med. Chem. 32: 437 (1989).

14. Peet, N.J. Heterocycl, Chem. 17: 1514 (1990).

15. For reviews, see: (a) Chem, Y Larock, R.C. In Modern Arylation Methods, Ackerman, J. ED Wiley/ VCH New York, PP401 (2009).

(b) Sanz, R.Org. Prep. Proced. Int. 40: 215 (2008).

16. (a) Lin, Z; Larock, R. C. J. Org. Chem. , 71: 3198 (2006).

(b) Lin, Z; Larock, R. C. Org. Lett. 5: 4673 (2003).

(c) Lin, Z; Larock, R. C. Org. Lett. 6: 99 (2004).

17. Lin, Z; Larock, R. C. J. AR.Chem. Sec. 127: 13112 (2005). 
18. Pintori, D. G Greaney, M.F.Org. Lett. 12: 168 (2010).

19. Yoshida, $\mathrm{H}$ Shirakawa, $\mathrm{E}_{4}$ Honda, $\mathrm{Y}$ Hiyama, T. Angew. Chem, Inted. 41: 3246 (2002).

20. Zhao, J Larock, R.C.J.Org. Chem., 72: 583 (2007).

21. Rogness D. C Larock, R.C. Tetrahedron Lett, 50: 4003 (2009).
22. Lin, Z; Larock, R.C.J. Org. Chem. 71: 3198 (2006).

23. M.A. Khan and S. Ahmad, Orient J. Chem., 28(2): 1061-1065 (2012).

24. S. Kumar, S. Yadav, S. Jadon, V. Kumar, A. M. Khedr and K.C. Gupta, Orient J. Chem., 28(4): 1833-1836 (2012). 